\title{
DESDE LAS ESCUELAS PRIMARIAS A LAS ESCUELAS SECUNDARIAS CAMPESINAS. LUCHAS POR LA EDUCACIÓN PÚBLICA EN CÓRDOBA, ARGENTINA*
}

\author{
Elisa Cragnolino ${ }^{1}$
}

\begin{abstract}
RESUMEN: El trabajo se refiere a las configuraciones de la educación rural en Córdoba, Argentina. Incluye una historización del sistema de educación primaria y la inclusión de las familias campesinas, proceso que nos parece importante recuperar para comprender luego las iniciativas de escolarización secundaria de un movimiento campesino. Sus prácticas educativas que se concretan en las "Escuelas Campesinas" se articulan en el marco de la "lucha por el territorio" frente al avance del agronegocio. Atenderemos a las condiciones que hicieron posible la elaboración del proyecto, su transformación y derivación en sedes de programas de terminalidad de secundaria estatal. Identificaremos actores, relaciones, sentidos disputados y tensiones que atraviesan estas singulares prácticas escolares.
\end{abstract}

Palabras claves: Políticas públicas. Escuelas primarias rurales. Educación secundaria campesina. Movimientos sociales.

\section{FROM ELEMENTARY SCHOOLS TO PEASANT SECONDARY SCHOOLS.

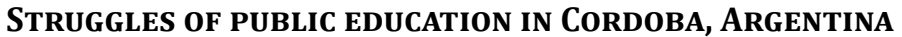

\begin{abstract}
This work addresses the configuration of rural education in Cordoba, Argentina. It includes the history of the primary education system and the inclusion of peasant families, a process that must be recovered so one understands the initiatives of secondary schooling developed by the movement. Educational practices implemented in Peasant Schools are articulated in the frameworks of "struggle for territory" and defense of peasant life versus the progress of agribusiness. We stuck to conditions that made possible the elaboration of the project, the transformation of proposal and its derivation in the headquarters
\end{abstract}

\footnotetext{
*Articulo resultado del Proyecto de Investigación "Transformaciones estructurales rurales, políticas públicas y disputas por educación”. Dirección: Elisa Cragnolino. Institución que acredita y financia: SECYT- Secretaría de Ciencia y Técnica de la UNC- Res Secyt 313/16 -Período 2016-2017. Se vincula también con el Proyecto de Voluntariado Universitario" Escuela Campesina como derecho". Secretaría de Politicas Universitarias de la Nación (Convocatoria 2015). Docente Responsable Elisa Cragnolino. Ejecutado desde 2016 y continúa. ${ }^{1}$ Universidad Nacional de Córdoba, Facultad de Filosofía y Humanidades, Centro de Investigaciones María Saleme de Burnichon - Córdoba, Argentina. E-mail: elisacragnolino@gmail.com DOI: 10.1590/ES0101-73302017177882
} 
of programs for secondary school termination. We also sought to identify actors, relationships, disputed senses, and tensions across these distinctive school practices.

Keywords: Public policies. Rural primary schools. Secondary peasant education. Social movements.

\section{DES ÉCOLES PRIMAIRES AUX ÉCOLES SECONDAIRES PAYSANNES. LES LUTTES DE L'ÉDUCATION PUBLIQUe À CóRdobA, ARgENTINE}

$R E S U M E$ : Ce travail fait référence aux paramètres de l'éducation rurale à Córdoba, en Argentine. On comprit l'histoire de l'enseignement primaire et de l'inclusion des familles rurales, un processus qui semble important de récupérer pour comprendre les initiatives que le mouvement développe dans l'enseignement secondaire. Les pratiques éducatives mises en œuvre dans les " écoles rurales " sont articulés dans le cadre de la " lutte pour le territoire " et de la défense de la vie paysanne avec l'avancement de l'agro-industrie. Nous nous appuyons sur les conditions qui ont rendu possible le développement du projet, la transformation de la proposition et sas dérivations dans les matrices des programmes par résiliation de l'école secondaire. On essaye d'identifier les acteurs, les relations, les significations contestées et les tensions qui traversent ces pratiques uniques scolaires.

Mots-clés: Politiques publiques. Écoles primaires rurales. L’enseignement secondaire des agriculteurs. Mouvements sociaux.

\section{Introducción}

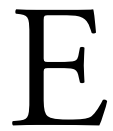

ste trabajo se refiere la configuración de la educación rural en el norte de la provincia de Córdoba, Argentina. Comenzaremos presentando la situación de la educación primaria, con una mirada de larga duración, para detenernos luego en los procesos de construcción e implementación de proyectos de escolarización secundaria que desarrolla un movimiento campesino.

Para comprender estos procesos recurrimos a perspectivas relacionales e históricas que reconocen la necesidad de vincular los procesos educativos con los estructurales, económicos y políticos. Entendemos que este desarrollo facilitará la comprensión de los procesos educativos actuales que se destacan por la presencia de un actor político y educativo emergente: el Movimiento Campesino de Córdoba (en adelante MCC). Sus iniciativas se configuran en el marco de la "lucha por el territorio" y la defensa de la vida campesina frente al avance del agronegocio. Reivindicando derechos, estas organizaciones avanzan en propuestas formativas que se concretan en las "Escuelas Campesinas" (EC). 
Atenderemos las condiciones que hicieron posible la elaboración del proyecto, su transformación y derivación en sedes de programas de terminalidad de secundaria estatal. Identificaremos actores, relaciones, sentidos disputados y tensiones que atraviesan estas singulares prácticas escolares que forman parte de la educación pública.

\section{Abordaje disciplinar y enfoques utilizados en el análisis de procesos de construcción de escuelas en territorios campesinos}

El enfoque que proponemos para las investigaciones que aquí presentamos corresponde a desarrollos de la Antropología de la Educación, con construcciones teóricas y metodológicas producidas dentro de la etnografía educativa en su vertiente latinoamericana (ROCKWELL, 2001). Estas recuperan, entre otras, las teorizaciones críticas gramscianas, las de la vida cotidiana de Agnes Heller, y las contribuciones de enfoques historiográficos contemporáneos que se inscriben dentro de la Historia Social y Cultural (E. P. Thompson, C. Ginzburg y R. Chartier). La etnografía analítica propone comprender las prácticas sociales a partir de la dimensión histórica, material y política de los acontecimientos; se señala la relativa autonomía y dependencia de los procesos locales respecto a los estructurales, la generación de prácticas sociales al interior de las instituciones concretas, los sujetos de estudio como protagonistas de la historia y como actores sociales con intereses personales e institucionales definidos (CALVO, 1992, p. 10). Con los elementos que aporta se puede repensar la noción de institución escolar, el de prácticas formativas, el lugar de los educadores y analizar lo político como proceso que atraviesa las tramas institucionales; estudiar la escuela y las prácticas desplegadas por múltiples actores; en este artículo, las organizaciones y movimientos campesinos, como parte de una sociedad civil donde la lucha política y la construcción de hegemonía se despliegan cotidianamente (ROCKWELL, 1987). Es posible además comprender las relaciones entre los espacios educativos (escolares y no escolares) y los procesos de producción cultural y reproducción social, identificando las prácticas de resistencia y las apropiaciones activas de los diferentes sujetos (ROCKWELL, 2009).

Desde estas perspectivas y poniendo atención en las disputas sobre prácticas y sentidos de la educación reconocemos en los movimientos sociales un actor de creciente significatividad, ya que a las exigencias de distribución del poder económico y de participación política se suma la disputa por la definición de qué es preciso enseñar y aprender, quién lo hace, de qué modo, en qué espacios.

En nuestro país, los estudios sobre el lugar de la educación en las luchas por la "recreación del campesinado" y la transformación de las relaciones sociales de esos territorios, son aún escasas, si se las compara con la amplia producción de Brasil. (Ejemplos de esta última son los artículos que se reúnen en este dossier). Entre los estudios de 
Argentina se destacan los trabajos de Norma Michi y su equipo de la Universidad de Luján. La autora, con un enfoque desde el materialismo cultural aborda el estudio de experiencias de educación de dos movimientos sociales del campo (MOCASE y MST). Se propone comprender la producción y reproducción cultural de estas organizaciones que intervienen en la disputa económica, política e ideológica y que al mismo tiempo gestan proyectos escolares que contrastan con la escuela regular y en este sentido participan de la construcción de proyectos de hegemonía alternativos (MICHI, 2010).

En todos los casos se trata de perspectivas relacionales e históricas que reconocen que la vida social condensa relaciones sociales, que se configuran como relaciones de poder y dan lugar a procesos de conflictos y resistencias.

\section{Las escuelas y la escolarización en el norte cordobés en el marco de las transformaciones estructurales y de las políticas educativas}

Argentina se ha destacado a nivel regional, por la amplitud del sistema de educación público y la obligatoriedad y gratuidad de las escuelas primarias que, tras la constitución del Estado Nacional y desde finales del siglo XIX, se crearon progresivamente en buena parte de su territorio. Si bien hay una amplia producción bibliográfica sobre estos procesos ${ }^{1}$, son relativamente recientes y en menor cantidad los estudios históricos sobre la educación rural y la mayoría de ellos se refieren a la región pampeana, siendo limitadas las investigaciones sobre la configuración de los sistemas de educación primaria en zonas campesinas.

Los estudios de Carli (1995), Lionetti (2007), Gutiérrez (2007), si bien están referidos a la pampa bonaerense y entrerriana, nos resultan de interés porque compartimos premisas teóricas y metodológicas que ligan la historia educacional con la historia económica, política y social rural. Estos trabajos señalan que las escuelas rurales, de dependencia provincial o nacional, se extendieron como parte del proyecto de la clase dirigente que a fines del siglo XIX diseñó un país que se integraría al mundo a partir de su economía agroexportadora. Este requería ocupación del espacio y fuerza de trabajo que aportarían los migrantes transatlánticos. Se pensó en las escuelas como instituciones que garantizarían el arraigo poblacional, su nacionalización y formación ciudadana y también el control y formación de los trabajadores. Los esfuerzos más importantes se realizaron en la órbita de la escuela primaria, encargada de la alfabetización primero y luego la educación básica.

Tal como hemos estudiado en nuestra tesis de Doctorado en Antropología, el sistema masivo de escolarización se hizo presente en Córdoba desde comienzos del siglo XX. Sobre todo, a partir de la década del 30, los Estados Nacional y Provincial crearon escuelas primarias, y lo hicieron aún en aquellas regiones consideradas marginales desde el punto de vista económico, como el norte, donde predominaba una población campesina (CRAGNOLINO, 2001) 
Veamos el proceso de transformación estructural que siguió uno de los departamentos de la región llamado Tulumba, en una mirada que abarca todo el siglo XX. El análisis fue realizado a través de fuentes estadísticas y "estudios en casos" ${ }^{2}$ con enfoque antropológico. La presentación histórica posibilitará la comprensión de la situación educativa actual y las prácticas desarrolladas por el MCC.

Enfocamos la atención en un territorio extra pampeano, que presentó desde los inicios del siglo pasado un desarrollo capitalista limitado y relativo, un medioambiente deteriorado debido a la tala indiscriminada de los bosques; el predominio de la producción para el mercado interno (fundamentalmente ganado) y el ser proveedor de mano de obra estacional para el agro pampeano.

El análisis estadístico y el relevamiento etnográfico ${ }^{3}$ permite reconocer el proceso económico y social que se observa en el Departamento Tulumba: en una primera etapa, 1900-1930, el predominio de explotaciones familiares menores a 100 has, con cierta diversificación productiva. Mientras en este período las familias aseguraban su reproducción directa a través de diferentes actividades prediales y la asalarización rural permitía sostener la unidad campesina, entre 1930-1970, la incorporación a los ciclos de la agricultura capitalista ya es insuficiente y la precariedad aumenta. Disminuye la tierra disponible y se reducen los rodeos. La descomposición hacia la dependencia laboral da paso a importantes procesos de descampesinización (MURMIS, 1992). Luego la situación se agrava con la caída de la demanda de trabajo rural en la zona pampeana por la mecanización de las cosechas. A esto se suma la incorporación de nuevas pautas de consumo, la entrada a la zona de productos y servicios que exigen una mayor monetarización y la fragmentación de los lotes, derivado de la herencia o venta de parte de la parcela y rodeos en épocas de escasez. En muchos casos estas crisis desembocan en la venta del predio.

Producida la retracción de la demanda de trabajo rural, con el desarrollo del mercado laboral urbano, industrial y de servicios y la implementación de políticas sociales que facilitan el asentamiento urbano, los destinos de las migraciones permanentes serán, las ciudades de Córdoba y Buenos Aires o pueblos de la zona.

Reflejarán este proceso los cambios observados en la concentración de la propiedad de la tierra, (desde la década del 60 aumentan las explotaciones de más de 1000 ha y sobre todo aquellas entre 2500 y 5000 has), la caída en la extensión de los rodeos caprinos, la disminución del personal ocupado en las explotaciones y el aumento en la proporción de trabajadores de la familia, que sin embargo también desciende como producto de las migraciones.

En la etapa siguiente: 1970 y hasta mediados de los 90, el estrechamiento de las bases de reproducción campesinas acentúa las tendencias emigratorias. La supervivencia se afecta por la pérdida de oportunidades de complementos salariales fuera de la parcela. Se profundiza así el proceso de descampesinización y desplazamiento hacia abajo. 
En la última etapa analizada, desde mediados de los 90 hasta la actualidad se han deteriorado aún más las condiciones de reproducción campesina. El avance del agronegocio afectó a esta zona con el corrimiento de la frontera agraria, la expansión del monocultivo ("sojización"), explotación degradante de los recursos naturales, concentración productiva, desplazamiento territorial de la producción campesina y conflictos por la tierra. Esta situación y el aumento de los intentos de expropiación dieron lugar al surgimiento de organizaciones campesinas que reivindican el derecho a permanecer en sus tierras, conservar modos de vida y acceder a la educación.

Veamos a continuación qué sucedió con la educación a lo largo del período analizado Las indagaciones estadísticas y documentales y las evidencias recogidas a través de análisis etnográficos ${ }^{4}$, muestran cómo a lo largo del siglo $\mathrm{XX}$ se abren establecimientos nacionales y provinciales, pero luego comienzan a cerrarse. Esto ocurre sobre todo a partir de la década del 50 cuando los pobladores abandonan el campo, se resienten las matrículas y algunas escuelas quedan sin alumnos. Sin embargo, en los momentos de incremento de la pobreza y salida con destino urbano, las familias campesinas no se habrían desvinculado de las escuelas, constituyéndose en agentes activos en su sostenimiento (CRAGNOLINO, 2001).

En 1914 existían en Tulumba 21 escuelas públicas, 5 eran elementales (hasta $4^{\circ}$ grado) y 16 rurales (hasta $2^{\circ}$ grado). En 1930 eran 33 las escuelas primarias, ninguna de las cuales era "graduada" (5 eran "elementales" y 28 "rurales"). En 1942 había 44 escuelas y solo 2 ofrecían la escolaridad completa 5 . Recién en 1949 el Ministerio de Educación de la Nación aprobó la extensión de la enseñanza hasta $6^{\circ}$ grado en su jurisdicción y a partir de 1954 la provincia implantó el ciclo completo en todas las escuelas primarias rurales.

A pesar de esta expansión del sistema educativo rural, la existencia de un establecimiento escolar en muchos de los parajes no implica para todas las familias el cumplimiento de la obligatoriedad escolar y la presencia regular de los niños. El interés por la escolarización se fue construyendo gradualmente conforme las familias advierten que los aprendizajes relativos al trabajo y el desempeño social transmitidos por ellas ya no eran suficientes y se hacía necesario incorporar a la escuela para garantizar, primero ciertos conocimientos, y luego una certificación, que los habilite para desempeñarse en el mundo laboral urbano (CRAGNOLINO, 2001, 2016).

Según el Censo Nacional de Población de 1980 la tasa de escolarización del departamento Tulumba en el grupo de 6 a 12 años alcanza el 92,3\%; en 1991 el $96 \%$ y en el 2001 al 98\%. Este crecimiento indica el reconocimiento de la imprescindibilidad de la educación primaria como instancia de asignación de oportunidades para la vida laboral y social. Y esto sucedería aún en estas zonas con predominio de hogares rurales, los que históricamente habían estado más ausentes del sistema. Claro que la carrera escolar finaliza para la mayoría de las familias en la escuela 
primaria y eso sucede en un momento histórico cuando en otras zonas rurales de la provincia, con un mayor desarrollo económico y sobre todo en las ciudades, se produce el acceso masivo a la escuela secundaria (CRAGNOLINO, 2001).

\section{Las escuelas secundarias rurales y las distancias materiales y sociales para las familias campesinas del norte cordobés}

La presencia de escuelas secundarias rurales o a aquellas ubicadas en los pueblos a las que podían acceder las familias campesinas, son en Tulumba, así como en el resto de los departamentos del norte cordobés, mucho más recientes que las primarias, que ya existían en la mayoría de los parajes. Por ejemplo, en 1972 se inaugura el ciclo básico de escuela secundaria en San José de La Dormida ${ }^{6}$, y luego de tres ańos se define la modalidad agropecuaria. Su existencia, sin embargo, no abrió nuevas oportunidades de acceso masivo para los hijos de las familias campesinas. A diferencia de lo que ocurre con la asistencia a las escuelas primarias rurales, durante las décadas del 70 y buena parte de los 80 , el ingreso de los jóvenes a la escolaridad secundaria no era objeto de previsión por parte de los padres campesinos, ni determinaba estrategias familiares para intentar concretarla. "La pobreza", la necesidad de que ayuden en el hogar, la chacra o con los animales; o la posibilidad de que se incorporen al mercado de trabajo como asalariados o jornaleros, los varones, y en servicio doméstico, las chicas; "las distancias" y la falta de medios de transporte, aparecen en forma recurrente para explicar las razones que los apartaron del nivel medio. Las condiciones objetivas son, sin duda, las determinantes. Sin embargo, mencionamos también las dificultades del "pasaje" a la secundaria urbana y las determinaciones que complejizan la resolución subjetiva de la salida de la casa.

Las escuelas secundarias se localizan en los pueblos y ciudades, pero también existió desde 1997 una oferta provincial "específica” para las zonas rurales: los Ciclos Básicos Unificados Rurales (CBUR). Estos corresponden al $3^{\circ}$ ciclo de la Educación General Básica, que la Ley Federal de Educación de 1993, había establecido como obligatorio. Surgen con la intención manifiesta de garantizar la asistencia de jóvenes rurales, "superar el fracaso escolar y deserción" y como "alternativa para la equidad y la atención a la diversidad" educativo urbano, y se ubican en una escuela primaria rural; comparten el espacio y en algunos casos esto es vivenciado como un conflicto. Están a cargo de un maestro tutor que proviene del Nivel Primario, tiene la mayor permanencia y va a ejercer la tutoría de disciplinas que son desarrolladas por los profesores, lo que implica para el maestro desempeñarse con contenidos más complejos y para lo cual no ha sido capacitado. Hay un importante ausentismo de los profesores disciplinares, cuyos salarios son insuficientes para cubrir los costos de traslado. Nos encontramos entonces con una institución con contenidos curriculares de Nivel 
Medio con tratamiento y cotidianeidad de Nivel Primario. Aparece como una instancia de formación pedagógicamente limitada y esto también lo señalan los padres (LIGORRIA, 2007). A estas debilidades se agrega el hecho de que, aunque se dice orientada a los jóvenes rurales, no contempla la realidad que implica para las familias la ausencia de los jóvenes y la necesidad que estas tienen de su trabajo y propone una asistencia regular de lunes a viernes; tampoco incorpora contenidos y saberes significativos para la realidad campesina.

En el año 2003 se abren en zonas rurales de la provincia de Córdoba los Ciclos de Especialización Rural (CER) que corresponden al último tramo de la escuela de nivel medio. Con la Ley de Educación Nacional (2007) este tramo será obligatorio. La nueva estructura del sistema que esta normativa propone incluye también "modalidades" entre las cuales se encuentra la Educación Rural. Este reconocimiento de la especificidad y una cierta "jerarquización”, con la creación de estructuras ministeriales específicas, políticas de formación docentes y recursos a través de programas, contrasta con la ley Federal de Educación, que ignoró a la educación rural y desmanteló la educación agrotécnica.

Sin embargo, entre otros actores, el MCC no parece reconocerse en estos proyectos y plantean la necesidad de construir escuelas alternativas a las oficiales. Según lo diagnostican, aunque algunas de las escuelas del campo han incorporado el tramo de la educación secundaria rural, parecen vivir al margen de los conocimientos producidos en y por la vida campesina.

\section{Proyectos alternativos de educación secundaria campesina y tensiones emergentes en su implementación}

El MCC, es una organización de $3^{\circ}$ grado constituida por seis "Centrales" que nuclean organizaciones zonales de $2^{\circ}$ grado, formadas a su vez por comunidades de base cuyas acciones se desarrollan en el norte de la provincia. Surge en el año 2004 y reúne aproximadamente a mil familias que participan en asambleas y a través de delegados. También a más de mil quinientas familias que se vinculan indirectamente a través de los proyectos que se promueven. Está integrado por campesinos y un grupo de "técnicos" de extracción universitaria que llegaron a fines de los 90 a los territorios, colaboraron con los diagnósticos y procesos organizativos iniciales y en la actualidad coordinan los programas educativos.

Las necesidades identificadas respecto a acceso al agua y al monte, diversificación productiva, venta de la producción a un "precio justo", atención de la salud, dieron lugar al desarrollo de prácticas organizativas orientadas a revalorizar formas de trabajo comunitario y solidaridad (MEIROVICH, 2015).

Las demandas por educación se plantean a poco de constituidas las organizaciones. Se articulan y no pueden entenderse desligadas de otros recla- 
mos derivados del avance del agronegocio y con afirmación de defensa territorial. Las reivindicaciones educativas surgen además en un contexto de luchas donde el MCC se va configurando como un actor político provincial, que participa de organizaciones a nivel nacional (Movimiento Nacional Campesino Indígena) e internacionales (Coordinadora Latinoamericana de Organizaciones del Campo y Vía Campesina). Se desarrollan inicialmente espacios de formación por comunidades, vinculados a cuestiones productivas, sectoriales y políticas, pero también se va construyendo un camino de formas pedagógicas y contenidos compartidos por las organizaciones nucleadas en Argentina, y orientadas por las prácticas de otros movimientos consolidados como el MST de Brasil.

Los reclamos por escolarización emergen con fuerza a partir del año 2007. Un diagnóstico reconoce que existe una gran cantidad de niños/jóvenes y adultos campesinos que no acceden a la educación media y un alto índice de deserción entre los que comienzan a cursar.

En el año 2007 había en los departamentos del Norte y Nor Oeste de la provincia 260 escuelas primarias rurales; los establecimientos de nivel medio rurales eran 38 y en estas últimas escuelas solo en 9 de ellas podía cursarse el nivel completo, ya que el resto ofrecía únicamente los primeros tres años (CRAGNOLINO et al., 2009).

Pese a la existencia de los CBUR y la creación luego de los CER, el MCC advertía que estos no estaban presentes en los parajes donde vivían la mayoría de las familias de las organizaciones y el Estado no aseguraba el traslado de los chicos. Agregaban que los dispositivos escolares creados, pese a nominarse "rurales", por su currículo y prácticas, "enseñaban para el desarraigo y pérdida de la identidad de los campesinos" (MNCI, 2012, p. 19). La exigencia de que se garantice una "escuela secundaria campesina en los territorios" se suma así a las luchas.

Desde el 2007 se dan dos procesos en paralelo. Por un lado, un trabajo interno y por otro aquel que se despliega respecto a la esfera gubernamental y las alianzas en el espacio público.

Como parte de las propuestas y experiencias de lo que se denomina en la orgánica del movimiento el "Área de Formación" durante las prácticas de "formación política" y en las reuniones comunitarias y de delegados, se comienzan a discutir los problemas en relación con la escolarización. Se advierte que "no poder seguir aprendiendo es una manera de desalojar a las familias campesinas... El proyecto de formación necesita espacios para nosotros y las nuevas generaciones que incluyan la emancipación como parte del curriculum" (MNCI, 2012, p. 97). Se avanza a partir de este reconocimiento de una necesidad y un derecho, con las discusiones acerca del "para qué" de la escuela y qué características debía tener.

Respecto a las acciones que se despliegan en la esfera gubernamental, el MCC reconoce como un hito la reunión con el Ministro de Educación de la 
Provincia de Córdoba. Durante este encuentro, los militantes campesinos dan a conocer sus demandas y plantean las características de la escuela que pretendían. El ministro habilita la posibilidad de que concrete la apertura de las EC, pero condiciona la aprobación a la presentación de "un proyecto de escuela".

Es en este momento cuando el MCC solicita apoyo la Universidad Nacional de Córdoba y comienza el trabajo de asesoramiento y acompañamiento de nuestro equipo, que implicó distintas actividades: En primer lugar, encuentros y discusiones con los integrantes del MCC para acordar los términos del requerimiento y características de la escuela. El trabajo colaborativo implicó relevamientos estadísticos que permitieron advertir los desfasajes entre la oferta y demanda por educación; recopilación y análisis de antecedentes normativos que reconocieran derechos; estudio de alternativas institucionales, organizacionales y pedagógicas existentes.

A partir allí se inicia un período de discusión y definición de la propuesta pedagógica curricular e institucional, que involucraba a los técnicos y a las bases de las organizaciones. La etapa de elaboración-consulta-discusión- reelaboración culminó con la escritura de un Proyecto a través del cual se propone la creación de tres escuelas campesinas de nivel medio, de alternancia, de gestión pública, cogestionadas con las familias de las comunidades y ubicadas en territorios del MCC.

En este proceso de elaboración de la propuesta fue también muy importante la articulación desplegada en relación con otras organizaciones no gubernamentales o colectivos más o menos institucionalizados que trabajaban desde la perspectiva de la "Educación popular"; en particular la Escuela de Agroecología del Movimiento Campesino de Santiago del Estero.

Finalmente, la presentación de la propuesta ante el Ministerio de Educación se produce en el mes de octubre del 2008 a través del inicio de un expediente. Una vez evaluado por la Secretaría de Educación el proyecto fue sometido a discusiones que se producían durante reuniones y derivado a diferentes "consultas técnicas", pero nunca fue aprobado.

Las dificultades, a nuestro entender tienen que ver con el estado de las correlaciones de fuerza existentes dentro del campo educativo. Veamos algunas de estas condiciones y procesos: En primer lugar, pese a que el propio ministro habilitó la posibilidad de planificar y poner en funcionamiento escuelas secundarias diferentes a las tradicionales, fueron observadas por los cuadros medios del ministerio, que son los que mostraron los mayores niveles de resistencia a aceptarlas. Este cuerpo burocrático, estaría dominado por la representación oficial de lo oficial (BOURDIEU, 1997) y la representación oficial de la escuela media supone una estructura que ellos mismos ayudaron a construir. Muchos de los funcionarios y miembros de los equipos técnicos que evaluaron la propuesta de EC, participaron en la construcción del CBUR, o fueron docentes y/o su- 
pervisores de esta "oferta específica" que el proyecto del MCC puso en cuestión. En este sentido estarían poco dispuestos a reconocer las limitaciones del sistema que ayudaron a crear.

En segundo lugar, este grupo de funcionarios y técnicos se presenta y distingue representando "el interés general de todas las familias" y "todos los alumnos" de Córdoba y, en virtud de esa representación, ayudan a imponer como legítimas las estructuras establecidas, las formas de gestión institucional y propuestas curriculares vigentes. Frente al "universalismo" de estas instituciones que sostienen, pero que no van acompañadas con la universalización de los medios que garanticen el acceso, destacan la excepcionalidad de la situación de los jóvenes campesinos. Al relegarlas al carácter de excepción le restan legitimidad al reclamo del MCC y limitan las posibilidades de aprobación (CRAGNOLINO, 2010).

Un tercer argumento esgrimido tiene que ver con "lo costoso" de la propuesta institucional y pedagógica que implica estructuras edilicias, nombramientos docentes por cargos concentrados en las escuelas, orientadores comunitarios que acompañen a los alumnos en los "tiempos de comunidad".

Habría además intereses corporativos poderosos vinculados al orden escolar establecido. Me refiero en particular a los profesores y su sindicato, que a través del a Estatuto del Docente, resguardan el derecho de acceso a los puestos de trabajo a través de un orden de mérito oficialmente establecido y no reconocen la posibilidad de que los padres de los alumnos y miembros de las organizaciones intervengan en la selección de docentes y directivo de las EC.

Otros actores del campo educativo que operan a nivel local y regional son los docentes y supervisores. La relación que el MCC ha tenido con ellos ha sido en algunas ocasiones conflictivas debido a una caracterización inicial que realizan, considerándolos como un sector uniforme, asociado solo con prácticas conservadoras, que en ocasiones se enfrenta con los padres y vecinos, no cumple con sus obligaciones de enseñar y hace poco por comprender las condiciones de vida y necesidades de las familias. Sin embargo, el MCC ha logrado progresivamente construir acuerdos con algunos de estos funcionarios en los territorios y sumarlos para sostener el proyecto de las EC.

A pesar de los pedidos de reconsideración ante el Ministerio de Educación y la búsqueda de apoyos ante diferentes sectores, entre ellos legisladores de la oposición, y aunque el proyecto estaría sustentado por las Leyes de Educación Nacional y Provincial vigentes que reconocen el derecho a la educación de los pobladores rurales, la necesidad de introducir medios para efectivizarla, la posibilidad de la alternancia y la gestión social, el MCC no logró la aprobación de las EC.

Sin embargo, en este transitar por las audiencias, y aprovechando la mayor flexibilidad que por su historia y características tiene la modalidad, se lograron acuerdos con la Dirección de Educación de Jóvenes y Adultos. Esta autorizó la 
apertura de tres sedes del Programa de Nivel Medio Semipresencial para Adultos, que funciona con tutorías que se concretan un día a la semana, cuando se reúnen los alumnos que se encuentran transitando los diferentes niveles y donde se trabaja a partir de módulos impresos definidos para un alumno universal urbano. Esto ha requerido un trabajo particular de los profesores del MCC para adecuar contenidos, abrir nuevas temáticas, incluir problemáticas relevantes para la realidad campesina y un análisis crítico del contexto.

Estas sedes, donde pueden cursar solo los mayores de 18 años, son unidades anexas a los Centros Educativos de Nivel Medio de Adultos, que funcionan en pueblos o ciudades cercanas; son gestionadas por técnicos del MCC que participan en calidad de Coordinadores Pedagógicos y Administrativos. Tienen en su planta docente a profesores orgánicos al MCC que accedieron a través de orden de mérito, pero también comparten trabajo con otros docentes sin vinculación con el MCC, que llegan a través de procesos de selección establecidos estatutariamente.

Desde hace cinco años los técnicos del MCC también gestionan en dos de las centrales un Programa de Inclusión y Terminalidad de Educación Secundaria para jóvenes de 14 a 17 ańos. Estos alumnos que no podían incluirse por su edad en el programa de Adultos ahora se incorporan al PIT, que funciona en las mismas sedes, articulados con escuelas regulares secundarias. Se proponen revertir el abandono del nivel, y para ello establece modos de cursada y estrategias pedagógicas menos rígidas que las de las escuelas regulares y más sensibles a las trayectorias y posibilidades vitales de los jóvenes.

\section{Tensiones que atraviesan las Escuelas Campesinas}

Hemos visto hasta aquí como surgieron las EC y la manera en que desde el MCC se fueron construyendo las posibilidades de garantizar espacios educativos para los militantes de las organizaciones, pero también para un conjunto de pobladores del campo, que se acercan atraídos por la cercanía física y la propuesta pedagógica. Se reelaboran los contenidos incluidos en los módulos oficiales o los presentes en los manuales escolares que se usan en las escuelas regulares de jóvenes, de modo de acercarlos al cotidiano y preocupaciones vitales de los pobladores del campo: agroecología, derecho a la tierra, acceso y recupero del agua, salud comunitaria, género.

Ahora bien, aunque con contenidos y dinámicas particulares y con una visión que articula la experiencia educativa con la participación comunitaria y política, las EC existen y funcionan en realidad, desde el momento en que se convierten en sedes de programas estatales. Esto implica para el MCC múltiples dificultades ya que tiene que conjugar un proyecto de escuela alternativo con las lógicas, requerimientos burocráticos administrativos y de gestión de las escuelas del sistema. 
Esto se hace evidente en distintas circunstancias: por ejemplo, cuando los docentes orgánicos del MCC tienen que convivir con profesores que llegan a la EC según un orden de mérito, pero que no comparten las propuestas político ni pedagógicas del MCC y pretenden que se cumpla con "lo establecido": el trabajo con módulos y la asistencia a requerimientos individuales de los alumnos. También en ocasiones en la que los docentes del MCC tienen que cumplir horarios en las instituciones de las que las $\mathrm{EC}$ son anexos, asistir a reuniones o seguir requerimientos administrativos sobre el rendimiento de los alumnos, o cuando son objeto de reclamos por los bajos niveles de cumplimiento de las metas establecidas.

Un segundo nudo de tensiones se observa con relación a las comunidades: en algunas de las centrales la demanda de abrir otras escuelas campesinas contrasta con el escaso número de militantes para acompañar este proceso. En efecto la escuela es reconocida como un espacio valioso y los integrantes de esas comunidades, ante la falta de respuesta estatal, se muestran dispuestos a impulsar la creación de una EC, pero el MCC no cuenta con "compañeros" suficientes para acompañar la gestión, apertura y sostener la dinámica cotidiana de nuevas EC.

Un tercer nudo de tensiones se da con relación a las familias y los estudiantes: en el caso del PIT surgen ciertas interpelaciones sobre lo que implica ser una escuela campesina diferente a las regulares. Esto se expresa de distinta manera: cuando los padres en ocasiones reclaman porque los chicos no cumplen con aquellas actividades o responsabilidades que corresponden al imaginario de una escuela regular (tareas, carpetas, evaluaciones; disciplina más estricta). También han aparecido exigencias de los alumnos respecto a contar con "la libreta escolar" (un documento donde se asientan los resultados de las evaluaciones) o que se nombre a aquellos que serán los portadores de banderas que inauguran cada acto de inicio o fin de clases o efemérides patrias.

\section{A modo de cierre}

Como hemos señalado, el interés de las familias campesinas del norte cordobés por la educación, que tiene en la actualidad expresión en las reivindicaciones y proyectos de las organizaciones del MCC, no son nuevas, en tanto es posible advertir una continuidad histórica en ciertas demandas educativas derivadas de prácticas que, aunque no reconocidas ni legitimadas por el sistema educativo, existieron.

En el norte cordobés, no hubo simplemente un "Estado Educador" que en determinados momentos decide la apertura o cierre de las escuelas. Estos movimientos siguieron los procesos de las transformaciones estructurales y en determinado momento supusieron para las familias campesinas el abandono del campo y la preparación de los hijos, a través de la escuela, para la migración y trabajo 
urbano. Además, las iniciativas estatales siempre fueron sostenidos por los padres de familias y vecinos, ya sea realizando trámites para la apertura de escuelas, cediendo tierras y locales para que funcionen, "haciendo beneficios" para mejorarlas. También estuvieron presentes resistiendo a través de distintas estrategias el cierre de las escuelas o la presencia de maestros que no acompañaban la educación de los hijos o las actividades comunitarias

A pesar de las continuidades observadas hay, sin embargo, en estas experiencias y procesos del MCC elementos "novedosos": en primer lugar, el MCC pelea para que los niños, jóvenes y adultos se eduquen no ya para salir del campo sino como condición para seguir reproduciéndose como campesinos. Reivindican la necesidad de construir y acumular conocimientos que permitan conservar su tierra, proteger el ambiente, mejorar las condiciones de vida y trabajo sin abandonar sus formas de existencia. En segundo lugar, mencionamos el hecho de que las familias campesinas hasta hace poco tiempo no se constituían como un actor colectivo; no se presentaban como una organización desde donde reclamar, negociar y discutir con los otros agentes del campo educativo. Existían demandas educativas y a menudo esto derivaba en reclamos ante las autoridades escolares; había disputas con los maestros o aquellos agentes educativos que no cumplían sus obligaciones, pero sus reclamos eran puntuales y locales. Finalmente, sus demandas no se articulaban con otros derechos relativos a la salud, el problema de la tierra y el agua, etc. Tampoco eran parte de un proyecto político alternativo, que es lo que sucede con la propuesta de EC; proyecto que se revitaliza en un contexto de avance del agronegocio y las expropiaciones de tierras.

En este trabajo reconstruimos el proceso que fue desde el diseño hasta la puesta en práctica de las EC. Advertimos las condiciones que imposibilitaron la aprobación del proyecto original que implicaba importantes rupturas en términos institucionales, organizativos, pedagógicos respecto a las escuelas secundarias regulares. Reconocimos las debilidades existentes, pero también cómo el MCC pudo aprovechar los intersticios que brindan ciertas dependencias ministeriales que han construido trayectos más flexibles y respetuosos de las trayectorias de los alumnos. Tanto los Programas de la Dirección de Adultos como aquellos dirigidos a Jóvenes de 14 a 17 años implicaron condiciones que facilitaron el desarrollo de las Escuelas Campesinas, aunque estas perdieran parte de las características reivindicadas originalmente en el proyecto, como la gestión comunitaria, el nombramiento de profesores y la alternancia. También pudimos ver como se construyeron alianzas con otros actores, organizaciones y colectivos que también buscan alternativas en la educación popular y lo que significó y posibilitó la articulación con la universidad.

Observamos también las tensiones que supone para el MCC desarrollar sus espacios formativos en el marco de los programas estatales. Hablamos de tensiones, pero al enumerarlas, podemos pensar en sí y cómo estas experiencias y 
sucesos que parecen contradecir la propuesta original, se convierte en oportunidades de nuevos desarrollos. Ser parte de programas estatales ha permitido sostener materialmente a los técnicos que viven de esos salarios; ha dado oportunidades de articulación con distintos agentes del Estado (en algunos casos inspectores regionales que permiten que una de las EC funcione en el edifico de una escuela primaria que ya no tiene alumnos o a la que sólo asisten unos pocos). También se han consolidado los vínculos construidos con los directivos de las escuelas secundarias regulares o de adultos, de las que son anexos. Del mismo modo han participado de reuniones con otros colegas que se encuentran gestionando los programas de jóvenes y adultos del sistema y podido compartir algunas de las experiencias innovadoras exitosas que desarrollaron; mostrar que las EC también tenían egresados y que algunos de ellos se han insertado en el nivel superior (estudiando profesorados o tecnicaturas). O que un grupo de alumnos del PIT ha sido reconocido a nivel provincial y nacional por el desarrollo de un biodigestor, con el que han sorteado diferentes instancias eliminatorias de una feria de ciencias que convoca a estudiantes de escuelas regulares.

La EC en estos casos deja de ser una escuela "limitada" y por lo tanto menos valiosa en tanto atiende a una población particular y pequeña, como lo señalaban los evaluadores del proyecto; deja de ser la "escuelita pobre para jóvenes y adultos pobres" y se convierte en un espacio de concreción de derechos a la educación, participación comunitaria y política y producción pedagógica

La existencia de EC en los territorios ha mostrado además a otras comunidades que carecen de escuelas propias, que es posible construirlas colectivamente. Aunque por el momento el MCC no pueda acompañar con sus técnicos las nuevas iniciativas, tiene la oportunidad de avanzar en la práctica de la organización política y ampliar su territorio de actuación con un trabajo que apunte a la emergencia de nuevas comunidades de base que se sumarían a partir de una reivindicación concreta ligada al derecho a la educación.

Finalmente, a pesar de los cuestionamientos que realizan las familias y los alumnos acerca de una dinámica escolar que poco se parece a la de la escuela regular, las intervenciones realizadas, estrategias desplegadas y conocimientos construidos, suponen la oportunidad de discutir el qué enseñar, el para qué de la formación de la escuela secundaria en el campo, el formato escolar y la definición de quienes pueden y deben participar en su construcción y sostenimiento.

\section{Notas}

1. Mencionamos solo a una de las autoras, entre muchos otros, Adriana Puiggrós que asumió la dirección y autoría junto con otros investigadores como Ossanna, Carli, De Luca, Gandulfo, Gagliano, Iglesias, Marengo, Rodríguez, Terigi, de una Historia de la Educación en la Argentina, que reúne diferentes tomos. Destacamos en particular los tomos 2, 3, 4 (PUIGGRÓS, 1991, 1992, 1993. 
2. Más que "estudio de casos" planteamos "estudios en casos" (GEERTZ, 1987), que expresen la direccionalidad histórica que está en juego en los procesos y relaciones investigadas.

3. Tulumba y buena parte del norte cordobés fue una zona "marginal" no sólo desde el punto económico y social, sino también historiográfico. Recurrimos entonces a una elaboración propia a partir de del relevamiento y análisis de una variedad de fuentes: Censos Nacionales de Población de 1914, 1947, 1960, 1970,1980, 1991 y 2001, para recuperar el desarrollo demográfico (distribución por sexos y edad, densidad, tasas de crecimiento, de migración). Los Censos Nacionales Agropecuarios (de 1908, 1937, 1960, 1969 y 1988), Censo Nacional Económica de 1994 e Informes Departamentales del Gobierno de Córdoba, Años 1991, 1993, 1996 hicieron posible reconstruir la evolución de la estructura agraria y el mercado de trabajo; identificamos cantidad, superficie y distribución de explotaciones agropecuarias; distribución diferencial de recursos y fuerza de trabajo, familiar y extrafamiliar en la producción y propiedad del ganado. Este análisis fue acompañado por "estudios en casos", para los cuales recurrimos a historias y relatos de vida, entrevistas en profundidad, observaciones, análisis de documentos personales de nuestros entrevistados (cartas de familiares migrantes a la ciudad). Para el análisis de procesos posteriores al 2001 que ya no forman parte de nuestra tesis, recurrimos a desarrollos de otros investigadores, en particular Hocsman y Preda (2005) y Hocsman (2009), para mencionar solo algunos. El acompańamiento a partir de proyectos extensionistas con el MCC nos permitió acceso a datos a través de fuentes documentales, observaciones y entrevistas en profundidad.

4. Las fuentes estadísticas utilizadas son: Anuarios Estadístico de la Provincia de Córdoba (AEPC) del año 1914, 1918, 1930 y 1942. Educación. Enseñanza Primaria 1960- 1962, Dirección General de Estadísticas, Córdoba, 1966. Estadísticas Educativas de los años 1970, 1976, 1983, 1994, Ministerio Educación Provincia Córdoba, entre otros. Los documentos consultados se encuentran en el Archivo del Ministerio de Educación de Córdoba y se trata de Actas de Consejo Provincial de Educación; Actas de Inspección Regional; Informes de directores de escuelas; entre otros. Para la reconstrucción etnográfica recurrimos a observaciones, relatos de vida, entrevistas en profundidad a diferentes actores que estuvieron y están presentes en la zona de estudio.

5. Ídem nota 4 .

6. "La Dormida", como es conocido este pueblo, se encuentra en la intersección de ruta nacional $\mathrm{N}^{\circ}$ 9 que conduce al norte del país y la ruta provincial $N^{\circ} 16$ que se dirige hacia una de las principales ciudades del norte cordobés. Se ha convertido desde hace aproximadamente cuatro décadas en el mayor centro poblacional, comercial y de servicios públicos del Departamento Tulumba, desplazando como centro económico, social y político, a la cabecera departamental (Villa Tulumba)

7. Fuente: CBU RURAL. Criterios para la organización del ciclo lectivo. Documento interno del Ministerio de Educación de la Provincia de Córdoba. Córdoba, 2000. (mimeo.).

\section{Referencias}

BOURDIEU, P. Razones prácticas: sobre la teoría de la acción. Barcelona: Editorial Anagrama, 1997. 
CALVO, B. Etnografía de la Educación. Nueva Antropología, México, v. 12, n. 42, p. 9-26, 1992.

CARLI, S. Entre Ríos. Escenario Educativo, 1883-1930. Paraná: UNER, 1995.

CÓRDOBA. Ministerio de Educación. CBU Rural: Criterios para la organización del ciclo lectivo. Documento interno. Córdoba, 2000.

CRAGNOLINO, E. Demandas y reivindicaciones educativas en familias campesinas. Trayectorias, disputas y tensiones en la construcción de escuelas alternativas. In: MANZANAL, M.; NEIMAN, G. (Orgs.) Las agriculturas familiares del Mercosur: trayectorias, amenazas y desafíos. Buenos Aires: Editorial Ciccus, 2010. p. 191-208.

. Educación y Estrategias de Reproducción Social: un estudio de casos en unidades domésticas de origen campesino. 595 p. Tesis (Doctorado en Antropología) - Facultad de Filosofía y Letras, Universidad Nacional de Buenos Aires, Buenos Aires, 2001.

. Las Escuelas Primarias en el campo Tulumbano. In: MUSIAL, G.B.S.; LIMA, S.F. (Orgs.). Histórias e memórias da escolarização em populaçóes rurais: sujeitos, instituiçôes, práticas, fontes e conflitos. Jundiaí: Paco Editorial, 2016. p. 449-488.

et al. Condiciones y alternativas de la educación rural obligatoria en el norte de Córdoba. In: FORO EDUCACIÓN RURAL, Córdoba, 14 set. 2009. mimeo.

GEERTZ, C. La interpretación de las culturas. México: Gedisa, 1987.

GUTIERREZ, T. Educación, agro y sociedad. Politicas educativas agrarias en la región pampeana: 1897-1955. Bernal: Universidad Nacional de Quilmes, 2007.

HOCSMAN, L. Expansión agraria, tierra y papel del Estado: análisis y reflexión sobre un caso argentino. In: FERNÁNDEZ, B.M. (Coord.). Movimientos Campesinos y Estado: Resistencias y Alternativas. Asunción: CLACSO, 2009.

HOCSMAN, L.; PREDA, G. Desarrollo agrario, estructura parcelaria y economía familiar en la provincia de Córdoba. In: JORNADAS INTERDISCIPLINARIAS DE ESTUDIOS AGRARIOS Y AGROINDUSTRIALES, 4. Buenos Aires: CIEA/FCE/UBA, 2005.

LIGORRIA, V. El Ciclo Básico Unificado Rural en el norte de Córdoba: ‘alternativa para la inclusión y el cumplimiento de la escolaridad obligatoria? In: CRAGNOLINO, E. (Org.). Educación en los espacios sociales rurales. Córdoba: Universidad Nacional de Córdoba, Editorial de la Facultad de Filosofía y Humanidades, 2007. p. 127-150.

LIONETTI, L. La misión politica de la escuela pública. Formar a los ciudadanos de la república (1870-1916). Buenos Aires: Miño y Dávila, 2007.

MEIROVICH, V. Por un territorio libre marchamos. Estudio sobre las prácticas comunicativas del Movimiento Campesino de Córdoba en el espacio público mediatizado. 203p. Tesis (Maestría en Comunicación) - Centro de Estudios Avanzados, Universidad Nacional de Córdoba, Córdoba, 2015.

MICHI, N. Movimientos campesinos y educación: el Movimiento de los Trabajadores Rurales Sin Tierra y el Movimiento Campesino de Santiago del Estero-VC. Buenos Aires: El Colectivo, 2010. 
MOVIMIENTO NACIONAL CAMPESINO INDIGENA (MNCI). Derecho a la educación Campesino indígena. Córdoba: Gráfica 29 de Mayo, 2012.

MURMIS, M. Tipología de pequeños productores campesinos en América Latina. In: PEON, C.E. (Org.). Sociología rural latinoamericana: hacendados y campesinos. Buenos Aires: CEAL, 1992. p. 79-117.

PUIGGRÓS, A. (Org.). Escuela, democracia y orden (1916-1943). Historia de la Educación en la Argentina. Buenos Aires: Galerna, 1992. Tomo 3.

(Org.). Historia de la educación en las provincias y territorios nacionales (18851945). Historia de la Educación en la Argentina. Buenos Aires: Galerna, 1993. Tomo 4.

(Org.). Sociedad civil y Estado en los orígenes del sistema educativo argentino. Historia de la educación en la Argentina. Buenos Aires: Galerna, 1991. Tomo 2.

ROCKWELL, E. Caminos y rumbos de la investigación etnográfica en América Latina. Cuadernos de Antropología Social, Buenos Aires, n. 13, p. 53-64, 2001.

La Experiencia Etnográfica: historia y cultura en los procesos educativos. Buenos Aires: Paidos, 2009.

- Repensando Institución: una lectura de Gramsci. Documento DIE. México: Departamento de Investigaciones Educativas, Centro de Investigación y de Estudios Avanzados del IPN, 1987.

Recibido en el 31 de marzo de 2017.

Aceito en el 01 de septiembre de 2017. 